\title{
Synthesis and Characterization of Sodium N,N'-dioctyl-ethylenediamine Diacyl Propionate
}

\author{
Jian Xu \\ College of Environmental and Chemical engineering \\ Tianjin Polytechnic University, Tianjin, China \\ Tel: 86-22-2452-8767 E-mail: xujianhappy66@163.com \\ Xiujun Liu (Corresponding author) \\ College of Environmental and Chemical engineering, \\ Tianjin Polytechnic University, Tianjin, China \\ Tel: 86-22-2452-8767Ｅ-mail: liuxiujun@tjpu.edu.cn \\ Bin Wang \\ College of Environmental and Chemical engineering, \\ Tianjin Polytechnic University, Tianjin, China \\ Tel: 86-22-2452-8767Ｅ-mail: wbjiaoshou@163.com

\section{Zhijun Qiao} \\ College of Environmental and Chemical engineering, \\ Tianjin Polytechnic University, Tianjin, China \\ Tel: 86-22-2452-8767_E-mail:qiaozhijun0928@163.com
}

\begin{abstract}
Sodium N,N'-dioctyl-ethylenediamine diacyl propionate, an anionic gemini surfactant, was synthesized by ethylenediamine with n-octyl bromide, followed by reaction with succinic anhydride, cesium hydroxide and triethylamine as catalyst, through $\mathrm{N}$-alkylation and N-acylation. Structure of the intermediate and final product was characterized by infrared spectrometer and nuclear magnetic resonance apparatus. The influences of material ratio and reaction time on the $\mathrm{N}$-alkylation product yield were investigated. Experimental results showed that the yield of the $\mathrm{N}$-alkylation product reached up to $64 \%$ under the optimum reaction conditions, the yield of the $\mathrm{N}$-acylation product was $39 \%$.
\end{abstract}

Keyword: Gemini surfactants, Characterization, Alkylation, Acylation

\section{Introduction}

Gemini surfactants, a new type of surfactant, are made up of two amphiphilic moieties connected at the level of the head groups or very close to the head groups by a spacer group [Zana R, 2002, p205-253]. Compared to traditional surfactants, gemini surfactants have many excellent performances for its special structure.Surface tension of water can be reduced effectively because of the low critical micelle concentration; and excellent synergistic effect can be performed when remixed with other surfactants[Menger F M, 2000, p1906-1920.]. At present the cationic Gemini surfactants are more reported in the literature [Yang Y., 2005, p67-77; Yu, L., 2008, p59-62.], while the anionic Gemini surfactants reported in the literature less[Skoog D A, 1992.].

Nowadays, the main problem hindering their applications in commerce is their complicated synthesis and purification processes. In this paper, the intermediate of the Gemini, N,N'-dioctyl ethylenediamine, was synthesized by 
ethylenediamine with n-octyl bromide at room temperature through $\mathrm{N}$-alkylation firstly. And then, an anionic gemini surfactant, Sodium N,N'-dioctyl-ethylenediamine diacyl propionate, was synthesized by the reaction of the intermediate with succinic anhydride. The influences of material ratio and reaction time on the $\mathrm{N}$-alkylation product yield were investigated. The cmc and $\gamma_{\mathrm{CMC}}$ of the Gemini were measured.

\section{Experimental}

\subsection{Materials and main instruments}

Materials: ethylenediamine, octyl bromide, $\mathrm{MF}, \mathrm{CsOH}, \mathrm{NaOH}$, tetrahydrofuran, succinic anhydride, triethylamine.

Main instruments: rotary evaporator; fourier transform infrared spectrometer; nuclear magnetic resonance apparatus; surface tension instrument.

\subsection{Synthesis method}

\subsubsection{Synthesis and purification of N, N'-dioctyl ethylenediamine}

The reaction mechanism is as follows:

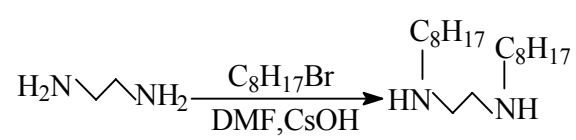

A certain mole ratio of ethylenediamine and n-octyl bromide was added to a certain amount of DMF containing CsOH. The mixyure was stirred for several hours at room temperature. White solid was obtained after filtration. The solid was washed with $\mathrm{NaOH}$ aqueous solution and distilled water several times, and then dried under reduced pressure and $50^{\circ} \mathrm{C}$ for 5-6 hours[Salvatore R N, 2002, p674-683.].

2.2.2 Synthesis and purification of Sodium N, N'-dioctyl-ethylenediamine diacyl propionate[Yoshimura T, 2005, p2682-2688.].

The reaction mechanism is as follows:

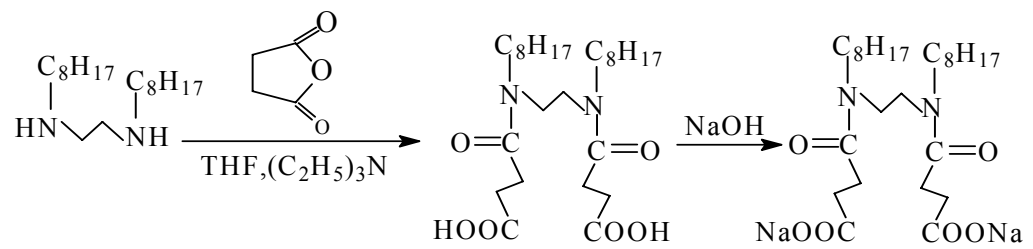

A certain amount of intermediate and an excess of succinic anhydride were added to tetrahydrofuran containing triethylamine. The reaction mixture was refluxed for several hours under stirring. After being cooled to room temperature, the solution was filtered to remove insoluble materials. The filtrate was evaporated under reduced pressure, the solid was washed with ethanol and distilled water for several times, dried under reduced pressure, and then turned into sodium salt after neutralization. The yield was $39 \%$.

\section{Results and Discussion}

\subsection{The effect of the material ratio on the $N$-alkylation product yield}

DMF $20 \mathrm{ml}$, CsOH $0.15 \mathrm{~g}$, ethylenediamine $0.01 \mathrm{~mol}$, reaction time was $24 \mathrm{~h}$, The material ratio was variable factor. The experimental results were shown in Figure 1. The yield of the intermediate was increased with the increasing of n-octyl bromide. When the material ratio was more than 2.75 , the yield decreased slowly. This was mainly because the intermediate transformed into tertiary amine. The optimum molar ratio was determined at 2.75.

\subsection{The effect of reaction time on the $N$-alkylation product yield}

Making the reaction time as variable factor, molar ratio 2.75:1, other reaction conditions were the same as 2.1. The results were shown in Figure 2. We can see that reaction time has great influence. The yield was increased with the prolonging of reaction time at the initial reaction stage, while reduced with the prolonging of reaction time. The reason may be as the prolonging of reaction time, the intermediate transformed into tertiary amine. The optimum reaction time was chose as $28 \mathrm{~h}$.

\subsection{FT-IR of the intermediate and the Gemini}

The FT-IR spectra of the intermediate were showed in Figure 3. The absorption peak of $3247.11 \mathrm{~cm}^{-1}$ was the stretching vibration of N-H. The absorption peaks of $2804.97 \mathrm{~cm}^{-1}$ to $2957.72 \mathrm{~cm}^{-1}$ were the stretching vibration of methyl and methylene groups. The peak of $752.99 \mathrm{~cm}^{-1}$ was the bending vibration of $\mathrm{N}-\mathrm{H}$. The absorption peaks of $1496.20 \mathrm{~cm}^{-1}$ and $1384.38 \mathrm{~cm}^{-1}$ were the symmetric and asymmetric bending vibration of methyl. The peak of $1133.08 \mathrm{~cm}^{-1}$ was the stretching vibration of C-N. 
The FT-IR spectra of the Gemini were shown in Figure 4. It could be find out that the FT-IR spectra of final product don't had the vibration of $\mathrm{N}-\mathrm{H}$. The absorption peak of $1729.68 \mathrm{~cm}^{-1}$ was the stretching vibration of carbonyl group.

\subsection{H-NMR spectra of intermediate and the Gemini}

1H-NMR spectra $\left(\mathrm{CDCl}_{3}\right)$ of intermediate were shown in Figure5. The constitutional formula of intermediate was as follows:

$$
-\underset{\mathrm{a}}{\mathrm{C}}-\underset{b}{\mathrm{NH}}-\underset{\mathrm{c}}{\mathrm{CH}_{2}}-\left(\underset{\mathrm{d}}{\mathrm{CH}_{2}}\right)_{6}-\underset{\mathrm{e}}{\mathrm{CH}_{3}}
$$

The date were shown in Figure5. The peak of chloroform proton was at the point of 7.28. The displacements of the other protons were a $(2.73 \mathrm{ppm}), \mathrm{b}(1.62 \mathrm{ppm}), \mathrm{c}(2.60 \mathrm{ppm}), \mathrm{d}(1.29-1.51 \mathrm{ppm})$, e $(0.89 \mathrm{ppm})$, consistent with theoretical value.

1H-NMR spectra $\left(\mathrm{D}_{2} \mathrm{O}\right)$ of Gemini were shown in Figure 6. The constitutional formula of Gemini was as follows:

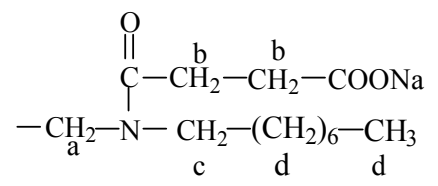

The date were shown in Figure6. The peak of $\mathrm{D}_{2} \mathrm{O}$ proton was at the point of 4.70 . The displacements of the other protons were a (3.94-4.01 ppm), b (2.44-2.46 ppm), c (3.02 ppm), d (1.03-1.12 ppm), consistent with target products.

\section{Conclusions}

An anionic gemini surfactant was synthesized, its surface activity was investigated. Optimum condition of N-alkylation was: DMF $20 \mathrm{ml}$; ethylenediamine $0.01 \mathrm{~mol}$; n-octyl bromide $0.0275 \mathrm{~mol}$; $\mathrm{CsOH} 0.15 \mathrm{~g}$, reaction time was $28 \mathrm{~h}$. Experimental results showed that the yield of the $\mathrm{N}$-alkylation product reached up to $64 \%$ under the optimum reaction condition, the yield of the $\mathrm{N}$-acylation product was $39 \%$.

\section{References}

Menger F M, Keiper J S. (2000). Gemini surfactants. Anger Chem. Int. Ed, 39:1906 - 1920.

Salvatore R N, Nagle A S, Jung K W. (2002). Cesium effect: High chemoselectivity in direct N-alkylation of amines. $J$. Org. Chem, 67: 674 - 683.

Skoog D A, Leary J J. (1992). Principles of instrumental analysis. Saunders College Pub.

Yang Y., Liu Y.B., Pu W. F. et al. (2005). Study advances of Gemini surfactants. Petroleum Geology and Recovery Efficiency, 12(6):67-70.

Yoshimura T, Nyuta K, Esumi K. (2005). Zwitterionic heterogemini surfactants containing ammonium and carboxylate headgroups.1.adsorption and micollization. Langmuir, 21 (7):2682-2688.

Yu, L., Sun, H. Q., Xiao, J. H. et al. (2008). Studies on the combination flooding with the Gemini carboxylates surfactant. Petroleum geology and recovery efficiency, 15(6):59-62.

Zana R. (2002). Dimeric and oligomeric surfactants. Behaviors at interface and in aqueous solution: a review. $A d v$. Colloid Interface Sci, 97: 205-253.

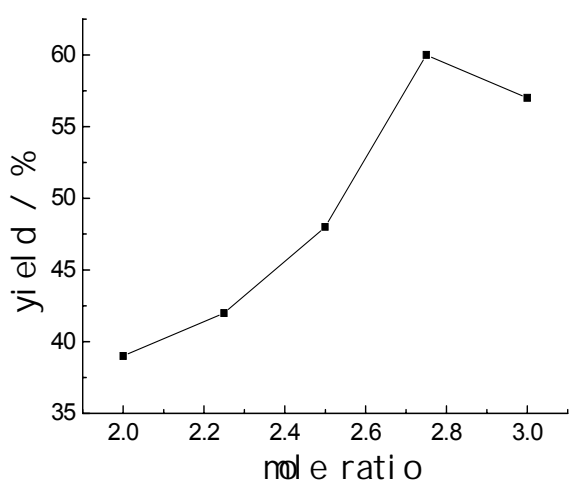

Figure 1. The effect of the material ratio on the N-alkylation product yield

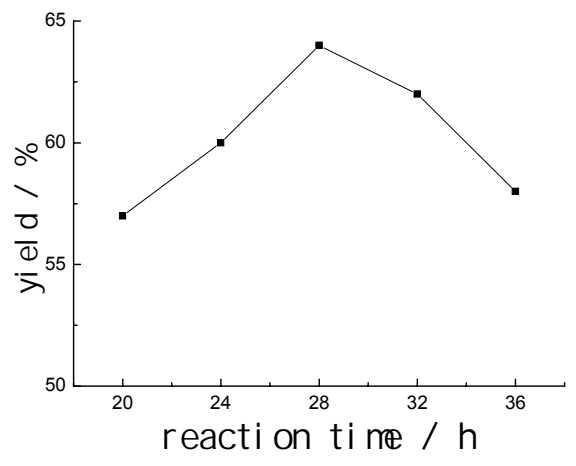

Figure 2. The effect of reaction time on the N-alkylation product yield 


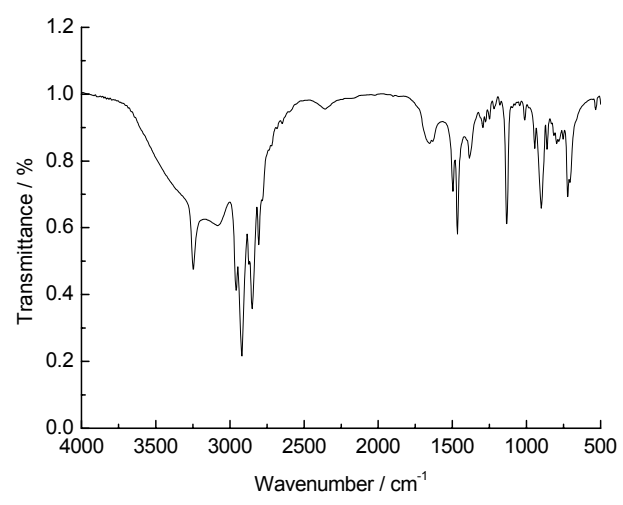

Figure 3. FT-IR spectra of the intermediate

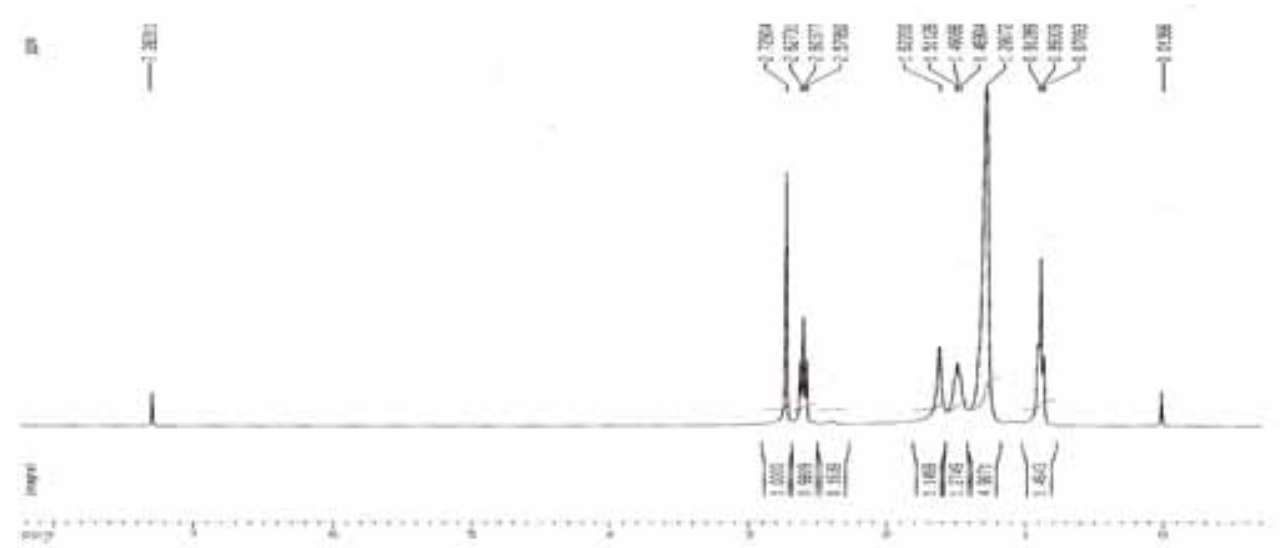

Figure 5. 1H-NMR spectra of intermediate

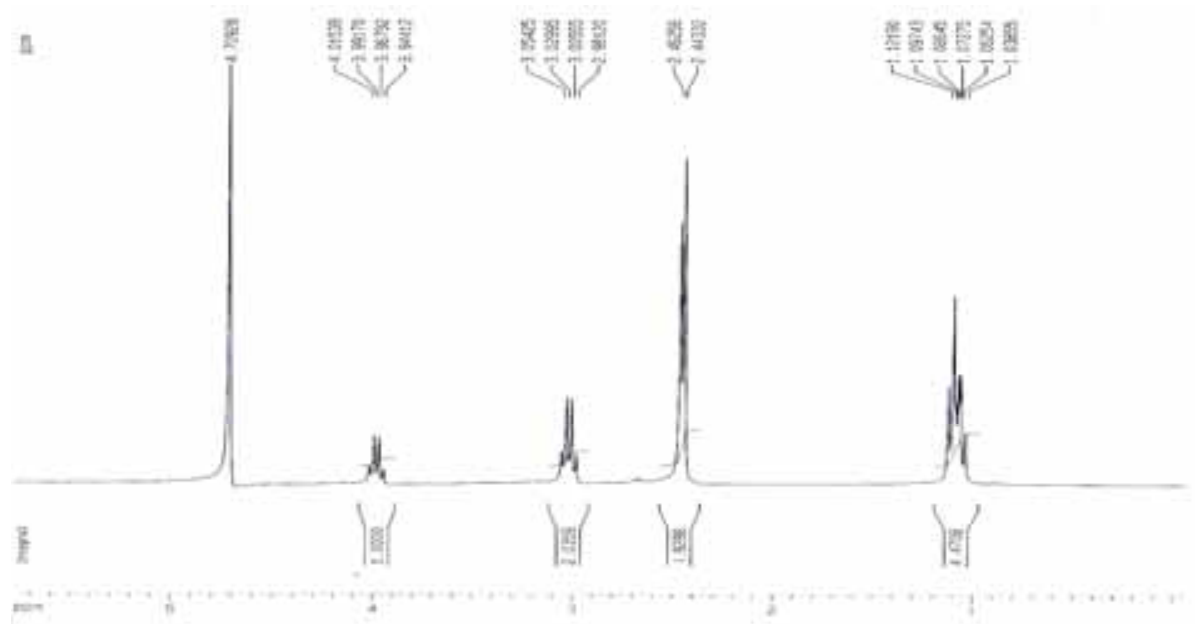

Figure 6. ${ }^{1} \mathrm{H}-\mathrm{NMR}$ spectra of Gemini 